\title{
Supportive care needs of people with pancreatic cancer: a literature review
}

\author{
Emma Scott, Anna Jewell
}

\section{Citation}

Scott E, Jewell A (2019) Supportive care needs of people with pancreatic cancer: a literature review. Cancer Nursing Practice. doi: 10.7748/cnp.2019.e1566

\section{Peer review}

This article has been subject to external double-blind peer review and has been checked for plagiarism using automated software

\section{Correspondence}

emmascottresearch@gmail.com

\section{Conflict of interest}

Emma Scott reports personal fees from Pancreatic Cancer UK to conduct the review. Anna Jewell reports grants to Pancreatic Cancer UK, with which she is affiliated, from Celgene UK, Mylan-BGP Products, NuCana, Shire, AngioDynamics, Erytech, Medapharma, Bristol-Myers Squibb, outside of the submitted work

\section{Accepted}

1 August 2018

\section{Published online}

January 2019

\section{Open access}

This is an open access article distributed under the terms of the Creative Commons AttributionNon Commercial 4.0 International (CC BY-NC 4.0) licence (see https:// creativecommons.org/licenses/ by-nc/4.0/), which permits others to copy and redistribute in any medium or format, remix, transform and build on this work non-commercially, provided appropriate credit is given and any changes made indicated

\begin{abstract}
Quality of life is crucial to people with pancreatic cancer due to poor survival rates and high symptom burden. Timely and person-centred supportive and palliative care can help people maintain a good quality of life. This article reports findings of a review that highlights the physical and psychological needs of people with pancreatic cancer and interventions that may help meet those needs. Information, supportive and palliative care needs should be assessed, and appropriate interventions implemented early in the illness and on an ongoing basis. The article also highlights the role of a multidisciplinary team, including clinical nurse specialists, oncologists, palliative care specialists, dietitians and mental health practitioners, in implementing personalised care.
\end{abstract}

\section{Author details}

Emma Scott, research consultant, Emma Scott Research Services, Haddenham, Buckinghamshire, England; Anna Jewell, director of operations, Pancreatic Cancer UK, London, England

\section{Keywords}

cancer, emotional aspects of care, emotional support, end of life care, palliative care, pancreatic cancer, patients, patient-centred care, patient outcomes, quality of life

\section{Background}

Pancreatic cancer is the 11th most common cancer in the UK with about 10,000 people diagnosed each year (Cancer Research UK (CRUK) 2018). People may not experience any signs or symptoms in the early stages, so the disease is often at an advanced stage when diagnosed. Late diagnosis and the small number of treatment options available means survival rates are low. In the UK, less than $7 \%$ of people with pancreatic cancer survive for five years (Allemani et al 2018) and it is the fifth most common cause of cancer death (CRUK 2018).

Late diagnosis usually causes a high symptom burden. Signs and symptoms may include abdominal and back pain, unexplained weight loss, indigestion, loss of appetite, changes in bowel habits, jaundice, nausea and vomiting, difficulty swallowing, recently diagnosed diabetes and psychological distress (Pancreatic Cancer UK 2016). Unfortunately, people with pancreatic cancer often continue to experience severe signs and symptoms to the end of life and, as a result, report poor quality of life (Lee et al 2012).

Given the often poor prognosis, supporting people with pancreatic cancer to maintain as good a quality of life for as long as possible is a vital aspect of their care (Lewis et al 2018). It is essential that they are supported to manage the signs and symptoms of the disease and treatment side effects throughout the care pathway. This can be achieved, in part, through effective and timely information and supportive care (Freelove and Walling 2006, Lee et al 2012, Gooden et al 2016). However, findings from National Cancer Patient Experience Surveys in England suggest that access to information and support is poorer for people with pancreatic cancer compared with other cancers (Quality Health 2016). For example, compared to the national average score people with upper gastrointestinal cancers, including pancreatic cancer, reported a lack of written information about the type of cancer they had (Quality Health 2016). It is therefore important to highlight the needs of people with pancreatic cancer and how those needs can be met.

The aims of the review were to:

» Outline what we know about the information, supportive and palliative care needs of people with pancreatic cancer.

॥ Explore the evidence on how these needs might be addressed.

» Highlight the gaps in knowledge on the information and supportive care needs of people with pancreatic cancer and discuss what further research is needed to fill those gaps. 
For the purpose of this review, palliative care encompasses symptom management, not just end of life care.

\section{Search strategy}

Searches of the PubMed, PsycInfo, CINAHL and Cochrane Library (Trials and Reviews) databases were carried out in March 2018 and covered literature published between January 2008 and March 2018. Search terms are listed in Box 1 and inclusion and exclusion criteria in Box 2.

Due to the small number of papers identified the quality of each was not assessed using an explicit tool - for example, Critical Appraisal Skills Programme checklists. However, the decision to include papers was based on Melnyk and Fineout-Overholt's (2011) levels of evidence (Box 3). Level 1 papers (systematic reviews and meta-analyses) provide the strongest, most comprehensive, evidence on a particular topic and level 7 papers (editorials, expert opinion and so on) the weakest (Melnyk and Fineout-Overholt 2011). As the search yielded such a small number of papers only level 7 papers were excluded from the review. A critique of the evidence is presented in the discussion.

\section{Box I. Search terms put into PubMed,} CINAHL, Psycinfo and Cochrane Library
Pancreatic cancer and
») Information needs
» Physical needs
») Psychological needs
》) Emotional support
\) Psychosocial needs
॥ Supportive care needs
») Supportive care interventions
\) Social support needs
») Practical support needs
I) Social care needs
») Follow-up needs
») Survivorship
॥) Palliative care needs
॥ Palliative care interventions

\section{Box 2. Inclusion and exclusion criteria}

Inclusion criteria

\) Published between 2008 and 2018

\) Pancreatic cancer-specific

\) Published in English

» Reported on the information and/or supportive/

palliative care needs of pancreatic cancer patients and/or interventions to meet needs

») Levels 1 to 6 on the levels of evidence hierarchy

\section{Findings}

The flowchart in Figure 1 shows the results of the literature searches. The review included 15 papers and the country of origin, aims and methods of each paper are shown in Table 1.

Findings from each paper eligible for inclusion were summarised and organised into common themes:

" High levels of supportive care needs experienced by people with pancreatic cancer.

» Needs change over time.

" Physical and psychological needs experienced by people diagnosed (including those with resectable, locally advanced and metastatic pancreatic cancer).

» How needs might be met.

\section{Findings}

High levels of supportive care needs experienced

Two recent papers explore specifically the needs of people with pancreatic cancer in Australia (Beesley et al 2016a, Beesley et al 2016b). The authors state no previous quantitative research has documented the supportive care needs of this population or their use of support services. Responding to the Supportive Care Needs Survey, a generic needs-assessment tool for cancer patients, participants reported high levels of need. Indeed, Beesley et al (2016a) refer to a 'tsunami of unmet needs' with $96 \%$ of respondents reporting some needs, while more than half had moderate-high unmet physical $(54 \%)$ or psychological $(52 \%)$ needs. The three most frequently reported moderatehigh needs were 'not being able to do things they used to' $(41 \%)$, 'concerns about the worries of those close' $(37 \%)$ and 'uncertainty about the future' $(30 \%)$. People with nonresectable (advanced) disease reported greater individual information needs, but overall their needs were like those with resectable disease.

Two thirds of respondents in Beesley et al's (2016a) study reported they were not provided with information about managing their illness and side effects. In particular, those with non-resectable disease needed more timely and clearer communication about their test results and how to manage the disease at home. Improved access to information resources enables emotional and physical preparation, for example for pain and dietary issues (Hagensen et al 2016). However, in Beesley et al's (2016a) study self-reported use of support was low and only $35 \%$ accessed information, $28 \%$ saw a dietitian, $18 \%$ used a complementary therapist and $15 \%$ consulted a mental health practitioner (Beesley et al 
2016b). Use of these services did not differ by whether people had a resection. However, palliative care access was significantly greater among those with non-resectable disease (59\% compared with $27 \%$ ).

\section{Needs change over time}

Using experience-based design techniques, Hagensen et al (2016) sought to understand the emotional content of the care experience to change practice. During the study they found that providers assumed patients and caregivers need as much information as possible at diagnosis but learned that processing information takes time and information provision should be tailored to meet the patient or caregiver needs.

Schildmann et al (2013) conducted a qualitative interview study to explore perceptions and preferences about information and treatment decision-making, and also found information needs and informationseeking behaviours change over time. When pancreatic cancer is diagnosed, trust in healthcare professionals takes precedence and patients often cannot, or do not want to, take part in treatment-related decisions. Over time, patients become more knowledgeable about their disease and treatment and take a more proactive role, for example asking more questions and becoming more involved in decisions about treatment (Schildmann et al 2013).

Beesley et al (2016b) conducted the first study to provide a longitudinal assessment of the needs of people with pancreatic cancer, exploring risk factors for current and future unmet supportive care needs. They highlighted that, overall, unmet needs are high and persist over time (nought to six months post-diagnosis and then two and four months later). However, data showed a reduction in needs over time for those who had a complete resection $(71 \%$ reported at least one need at baseline, dropping to $63 \%$ four months later) and an increase

\begin{tabular}{|c|}
\hline $\begin{array}{l}\text { ॥ Level } 1 \text { Systematic review and meta-analysis of } \\
\text { randomised controlled trials; clinical guidelines based } \\
\text { on systematic reviews or meta-analyses } \\
\text { ॥ Level } 2 \text { One or more randomised controlled trials } \\
\text { ॥ Level } 3 \text { Controlled trial (no randomisation) } \\
\text { 》" Level } 4 \text { Case-control or cohort study } \\
\text { ॥ Level } 5 \text { Systematic review of descriptive and } \\
\text { qualitative studies } \\
\text { ॥ Level } 6 \text { Single descriptive or qualitative study } \\
\text { 》 Level } 7 \text { Expert opinion }\end{array}$ \\
\hline
\end{tabular}

in needs for people with locally advanced disease $(73 \%$ reported at least one need at baseline, increasing to $85 \%$ four months later) and metastatic disease (66\% reported at least one need at baseline, increasing to $88 \%$ four months later).

Depression and pain were associated significantly with physical needs reported during the first six months post-diagnosis. Experiencing pain and having locally advanced disease were the main factors associated with future physical needs (needs reported at two and four-month follow-up) possibly because these patients were not accessing palliative care, for example for symptom management (Beesley et al 2016b). Anxiety, depression and pain were associated with psychological needs reported during the first six months post-diagnosis.

Figure I. Flowchart showing literature search outcome

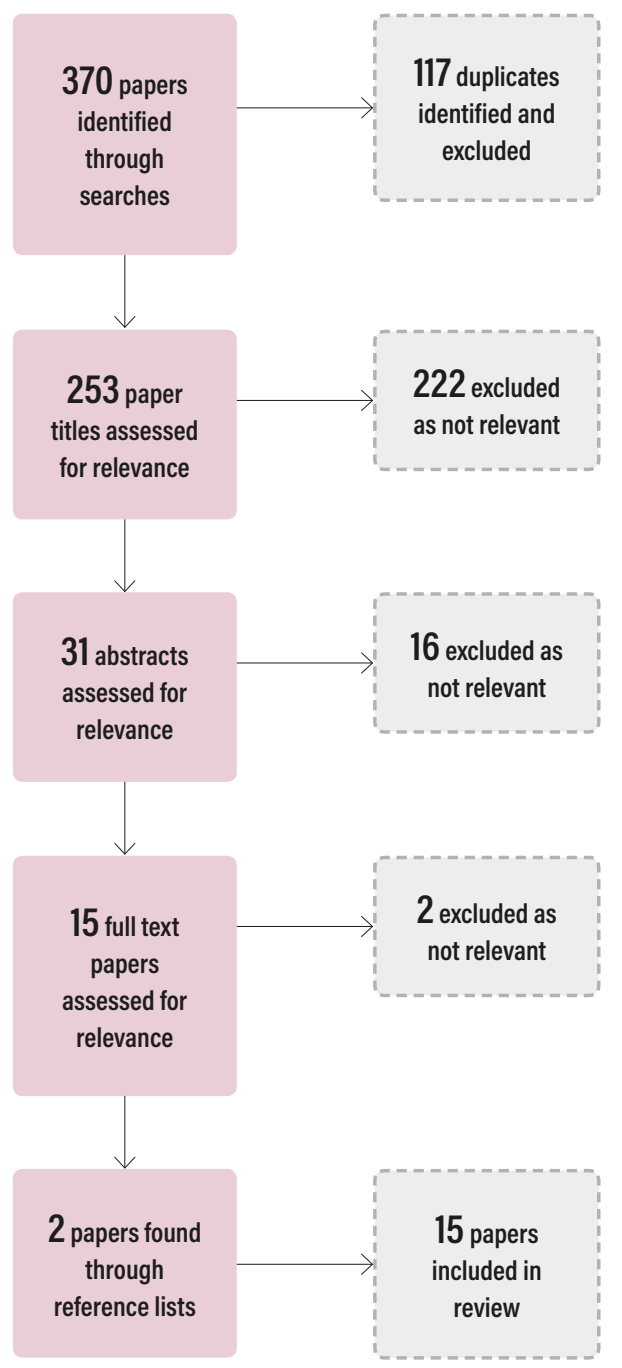

\section{Key points}

- Pancreatic cancer is often at an advanced stage when diagnosed. Late diagnosis can cause a high symptom burden

- Supporting people with pancreatic cancer to maintain as good a quality of life as possible for as long as possible is a vital aspect of their care

- To meet people's needs it is important to explore and evaluate new models of care, such as early referral to supportive and palliative care and closer integration of oncology and palliative care

\section{Permission}

To reuse this article or for information about reprints and permissions, please contact permissions@rcni.com 


\section{Table 1. Papers included in the review continued}

\begin{tabular}{|c|c|c|c|c|}
\hline Authors & Year & Country & Type of paper & Aims \\
\hline $\begin{array}{l}\text { 1. Schenker } \\
\text { et al }\end{array}$ & 2018 & US & Research & $\begin{array}{l}\text { Assess the feasibility, acceptability and perceived effectiveness } \\
\text { of early specialty physician-led palliative care for patients with } \\
\text { advanced pancreatic cancer and their caregivers }\end{array}$ \\
\hline
\end{tabular}

\begin{tabular}{|c|c|c|c|c|}
\hline 2. Frick et al & 2017 & US & Research & $\begin{array}{l}\text { Describe treatment patterns and related side effects, surveillance } \\
\text { care patterns and perceptions of a survivorship care plan tool }\end{array}$ \\
\hline 3. Beesley et al & $2016 a$ & Australia & Research & $\begin{array}{l}\text { Describe people's needs and use of support services and examine } \\
\text { whether these differ according to whether patients had surgical } \\
\text { resection or not }\end{array}$ \\
\hline 4. Beesley et al & $2016 b$ & Australia & Research & $\begin{array}{l}\text { Determine if the supportive care needs of people with pancreatic } \\
\text { cancer change over time and identify factors associated with } \\
\text { current and future unmet needs }\end{array}$ \\
\hline 5. Sun et al & 2016 & US & Research & $\begin{array}{l}\text { Determine the feasibility of an interdisciplinary supportive care } \\
\text { planning intervention in patients with pancreatic cancer during } \\
\text { treatment }\end{array}$ \\
\hline $\begin{array}{l}\text { 6. Hagensen } \\
\text { et al }\end{array}$ & 2016 & US & Research & $\begin{array}{l}\text { Understand the emotional content of the care experienced by } \\
\text { pancreatic cancer patients }\end{array}$ \\
\hline 7. Gooden et al & 2016 & Australia & $\begin{array}{l}\text { Non-systematic } \\
\text { literature review }\end{array}$ & $\begin{array}{l}\text { Explore the need for integrated psychosocial and supportive care } \\
\text { for patients with pancreatic cancer }\end{array}$ \\
\hline 8. Muircroft & 2016 & Australia & $\begin{array}{l}\text { Non-systematic } \\
\text { literature review }\end{array}$ & $\begin{array}{l}\text { Australian perspective on treatment of patients with pancreatic } \\
\text { cancer, supportive care and future directions for management }\end{array}$ \\
\hline 9. Grant et al & 2015 & US & Research & $\begin{array}{l}\text { ॥ How many people visit a webpage where they can interact with } \\
\text { a palliative care nurse practitioner } \\
\text { ॥ See how many would ask questions } \\
\text { ॥ Determine the type of questions } \\
\text { ॥ Obtain feedback on usefulness }\end{array}$ \\
\hline
\end{tabular}

\begin{tabular}{|c|c|c|c|c|}
\hline $\begin{array}{l}\text { 10. Engebretson } \\
\text { et al }\end{array}$ & 2015 & US & Research & $\begin{array}{l}\text { Evaluate patient and caregiver perceptions of diagnosis and daily } \\
\text { life with pancreatic cancer }\end{array}$ \\
\hline
\end{tabular}

\begin{tabular}{|l|l|l|l|}
$\begin{array}{c}\text { 11. Polakowski } \\
\text { et al }\end{array}$ & 2015 & US & $\begin{array}{l}\text { Case study and } \\
\text { non-systematic } \\
\text { literature review }\end{array}$ \\
$\begin{array}{c}\text { 12. Gooden and } \\
\text { White }\end{array}$ & 2013 & Australia & Research \\
\hline $\begin{array}{c}\text { 13. Grant and } \\
\text { Wiegand }\end{array}$ & 2011, & US & Research \\
\hline $\begin{array}{c}\text { 14. Schildmann } \\
\text { et al }\end{array}$ & 2013 & Germany & Research \\
\hline $\begin{array}{c}\text { 15. Torgerson } \\
\text { and Wiebe }\end{array}$ & 2013 & US & $\begin{array}{l}\text { Non-systematic } \\
\text { literature review }\end{array}$ \\
\hline
\end{tabular}

Outline risk factors, diagnosis and prognosis, treatment and survivorship care planning is also demonstrated

Understand how pancreatic cancer is experienced from the perspective of those diagnosed and the carer/family to identify areas of unmet psychosocial supportive care needs

Identify the palliative issues of those accessing a web page where they could interact with a palliative care nurse practitioner and determine the type of responses the nurse practitioner provided

Explore pancreatic cancer patients' perceptions of, and preferences for, information and treatment decision-making

Highlight the problems faced when caring for patients with advanced pancreatic cancer. Summarise issues facing patients and families along the disease trajectory potential complications of pancreatic cancer. The importance of

\section{Methods}

॥ Mixed-methods pilot randomised control trial

I) Patient-caregiver pairs were randomised (2:1) to receive specialty palliative care in addition to standard oncology care, compared with standard oncology care alone

॥ $n=30$

॥ Quantitative

॥ Convenience sample from pancreatic cancer survivors and their proxies voluntarily using a web-based tool for creating survivorship care plans

) $n=117$

I) Quantitative

॥) Supportive Care Needs Survey

I) $n=136$

》) Quantitative

I) Supportive Care Needs Survey

1) Self-administered at nought to six months post-diagnosis $(n=116)$ then follow-up two $(n=82)$ and four months $(n=50)$ later

I) Prospective, pre- and post-intervention pilot study

I) $n=10$

\) Qualitative

») Experience-based design

Not applicable (N/A)

N/A

॥) Mixed-methods descriptive design

》) $n=2,174$ visits to the webpage

॥ $n=84$ participants sent 110 questions/ comments

I) The Pancreatic Cancer Action Network administered a 25-minute online survey

I) $n=397$ respondents, including 184 patients and 213 caregivers

I) Case study

I) $n=1$

॥ Qualitative inquiry framework

॥ $n=35$

॥ Qualitative descriptive approach

I) $n=48$ participants posted 55 questions to the palliative care nurse practitioner

\) Qualitative interview study

I) $n=12$

N/A 
In addition, those with metastatic disease had higher odds of future psychological needs. The authors suggest people with metastatic disease are at greater risk of experiencing unmet needs over time due to faster disease progression, more symptoms and fear of death. Beesley et al (2016b) conclude that addressing pain and psychological distress is vital to meeting the significant unmet needs of people with pancreatic cancer.

\section{Physical needs}

Some of the literature highlights in greater detail the physical needs experienced by people with pancreatic cancer. Lack of energy, tiredness, pain, feeling unwell and work around the home were unmet needs at moderate to high levels in Beesley et al's (2016a) study. The authors argue fatigue and pain affect quality of life and that meeting these needs should be a priority.

A pilot qualitative study, which supports Beesley et al's (2016a) findings, explored the palliative care issues raised by patients and their families accessing a webpage where they could ask a nurse practitioner questions. The focus of questions was physical aspects of pancreatic cancer, in particular signs and symptoms of the illness or adverse side effects of treatment. The most common issue was pain followed by eating/nutrition and adverse effects of medication or treatment (Grant and Wiegand 2011, Grant and Wiegand 2013).

In a review of the supportive care needs of patients with pancreatic cancer, Muircroft (2016) outlines how weight loss can be a significant issue. This can arise through various causes including cachexia/anorexia, pancreatic exocrine insufficiency, side effects of surgery, chemotherapy and radiotherapy, nausea and vomiting and diabetes.

Muircroft (2016) suggests that management of cachexia needs a multidisciplinary approach, including dietitians and physiotherapists. Permanent taste alteration due to chemotherapy can also contribute to weight loss as it alters the appeal of food or supplements. This can affect compliance with oral nutritional supplements and dietary intake and therefore needs monitoring during and after chemotherapy (Muircroft 2016).

For people treated for resectable or nonresectable disease, pancreatic exocrine insufficiency, not producing or secreting enough digestive enzymes from the pancreas for adequate digestion, is common. It can affect quality of life significantly by causing weight loss, malnutrition, steatorrhoea, pain and bloating, which can subsequently make people fearful of leaving the house (Gooden and White 2013). Exocrine insufficiency requires treatment with the oral administration of pancreatic enzymes - pancreatic enzyme replacement therapy (PERT).

In Gooden and White's (2013) qualitative inquiry into the psychosocial supportive care needs of people affected by pancreatic cancer a quality of life theme was 'difficulty in managing gut symptoms and complex dietary issues'. They highlighted a lack of information on managing symptoms of pancreatic exocrine insufficiency exacerbated by a lack of routine dietary consultation and poor understanding of dose-to-diet guidelines for taking PERT.

The researchers concluded that management of pancreatic exocrine insufficiency is an area of unmet need that severely affects quality of life and increases carer burden. They suggested that exocrine insufficiency is ideally managed in a multidisciplinary setting involving a dietitian, clinical nurse specialist (CNS) and palliative care practitioners.

\section{Psychological needs of people with}

\section{pancreatic cancer}

Several studies and literature reviews have explored the psychological effects of pancreatic cancer. Distress (depression and anxiety) occurs more frequently in people diagnosed with pancreatic cancer than other cancers (Torgerson and Wiebe 2013, Gooden et al 2016) and figures range from $33 \%$ to $70 \%$ of patients experiencing depression at some point in the disease trajectory (Torgerson and Wiebe 2013).

In Beesley et al's (2016a) Australian study $90 \%$ of respondents reported at least one psychological need. They reported unmet needs around family anxieties, uncertainty about the future, sense of personal control and positive adjustment. Only $3 \%$ reported having all their psychological needs met and $76 \%$ reported having at least one, but not all, of their psychological needs met. However, little psychological support was accessed with only $16 \%$ consulting a psychologist, psychiatrist, social worker or telephone counsellor.

Beesley et al (2016a) speculate some needs may have been met through using informal support from family and friends.

In a study exploring the questions people asked a palliative care nurse on a website the largest subgroup of palliative care issues raised was psychological concerns, most of which related to anxiety about the illness (Grant et al 2015). Likewise, findings from an online survey evaluating perceptions of diagnosis and daily life with pancreatic cancer identified the 
'enormous psychological toll' of the disease (Engebretson et al 2015).

In their review exploring the need for integrated psychosocial and supportive care for people with pancreatic cancer, Gooden et al (2016) discuss how nihilism affects patients, their families and healthcare professionals, and draw attention to the problem of 'therapeutic nihilism' where healthcare professionals lack belief in the value of an intervention. Gooden et al (2016) stress that there is potential for this nihilism to affect care provision, for example poor coordination of care with, or referral to, other specialties and lack of communication about end of life. From patients' perspectives, experiencing this nihilism can lead to increased 'existential distress', which may include hopelessness, demoralisation, loss of dignity and control, and feeling a burden to others (Gooden et al 2016).

It is thought that early detection and treatment of distress is required through routine screening and access to psychiatric services (Muircroft 2016). Gooden et al (2016) also call for referral to 'meaning-centred' therapies to explore issues including loss of hope and purpose in life and meaning in life, the existential questions that affect some people with pancreatic cancer.

Interventions tested to meet needs Interventions that have been tested to see whether they help meet the information, supportive and palliative care needs of people with pancreatic cancer include integrating palliative care within oncology, holistic needs assessments, survivorship care plans and education sessions. For example, on physical and psychological concerns.

\section{Early referral to palliative care and integration within oncology}

Due to poor survival rates and the high symptom burden experienced by people with pancreatic cancer, palliation of pain and nonpain symptoms is crucial. Discussion about and referral to supportive and palliative care, including hospice care, should be offered early, for example from the point of diagnosis (Torgerson and Wiebe 2013, Beesley et al 2016b, Gooden et al 2016, Muircroft 2016). In addition to physical symptom management, the advantages of early referral to palliative care include earlier access to psychosocial support, care coordination, referral to different specialties, for example dietitians, and preparation for end of life (Muircroft 2016). Early referral also allows people to build relationships with supportive and palliative care teams as the disease progresses. Late referral could result in patients and their significant others having to get to know a new team during a period of crisis or transition, which could increase distress (Torgerson and Wiebe 2013).

Integrating palliative care in oncology is one model that might help ensure early referral. Equally, palliative care specialists can complement the work of oncology teams (Muircroft 2016). Schenker et al (2018) conducted a pilot randomised control trial to explore the feasibility, acceptability and effectiveness of early specialty physicianled palliative care for people with advanced pancreatic cancer. Findings suggest that integrating palliative care in oncology clinics may increase acceptability and perceived effectiveness. The intervention included faceto-face visits with a palliative care physician. Schenker et al (2018) describe how the visit content focused on relationships and rapport building, illness understanding, preferences and concerns, patient and caregiver needs, including physical symptoms, emotional distress and social/financial caregiver burden. An email was sent before and after each visit to encourage oncologist-palliative care communication about changes in patients' symptoms, functioning, distress or care goals.

Positive experiences reported by patients included gaining emotional support, information about cancer and treatments, symptom management and talking about the future and what to expect (Schenker et al 2018). Negative experiences included the inconvenience of too many medical appointments/spending too much time at hospital, having to think about difficult scenarios such as the severity of the illness and no perceived palliative care needs.

Indeed, for patients with fewer needs the role of palliative care was unclear. Physicians recommended embedding palliative care in oncology clinics, for example conducting a review at a multidisciplinary team (MDT) meeting, tailoring services to patient needs, for example looking at frequency and content of sessions, and supporting face-toface communication between oncologists and palliative physicians to further improve the service. Palliative care physicians also recommend incorporating nurse-led palliative care to increase the availability of palliative care services. They suggest these improvements might increase acceptance of palliative care among patients and ensure they do not receive mixed messages about prognosis or treatment goals (Schenker et al 2018). 


\section{Holistic needs assessments and} survivorship care plans

Physical and psychological needs experienced by people with pancreatic cancer persist and change over time (Beesley et al 2016b). To ensure people receive timely, appropriate and individualised support, their needs must be assessed and on an ongoing basis.

Recent National Institute for Health and Care Excellence (NICE) guidelines (2018) for the diagnosis and management of pancreatic cancer in adults outline the importance of psychological support. People should have access to psychological support throughout their care pathway and support should assess the psychological effects of fatigue, pain, gastrointestinal symptoms, including changes to appetite, nutrition, anxiety and depression that may affect their daily lives. Support should be available on an ongoing basis, relevant to the stage of the person's condition and tailored to their needs (NICE 2018). However, the guidelines do not suggest specific support interventions.

Holistic needs assessments and care plans have been trialled as ways of identifying patient information and support needs. Holistic needs assessments help to highlight the most important issues for the person affected by cancer at that time and can then inform the development of a care plan with a CNS. The assessment ensures people's needs are met in a timely and appropriate way (Macmillan Cancer Support 2013).

Survivorship care plans aim to improve communication between healthcare professionals and patients. Frick et al (2017) conducted a quantitative study to explore treatment patterns, side effects, surveillance care plans and perceptions of a survivorship care plan tool. Only $5 \%$ of people with pancreatic cancer who were surveyed received survivorship information following treatment.

Of the people who responded to a brief survey at the time they received their care plan, $83 \%$ said they would share it with their healthcare team, although 50\% wanted more information than the plan provided (Frick et al 2017). In terms of the information patients may want to see in a survivorship care plan, Polakowski et al (2015) argued there is a significant need for information that supports people to detect side effects/ complications of treatment and assess signs and symptoms to manage the effects of pancreatic cancer. The aim of the plan should be to improve care coordination and patient satisfaction which gives patients a sense of comfort as they have a plan for tackling the disease (Polakowski et al 2015).

\section{Education sessions}

An 'interdisciplinary supportive care planning intervention' for people with pancreatic cancer was developed and feasibility-tested by Sun et al (2016). Here too the role of the CNS is important. CNSs conduct a quality of life assessment, present this to the MDT and run education sessions (Box 4).

During a pilot of the intervention, discussions in the MDT and educational sessions focused largely on physical and psychosocial concerns. In the educational sessions patients were provided with written information and asked to identify priority areas for discussion. The sessions were conducted face-to-face or by telephone and focused on the areas outlined in Box 4.

\section{Box 4. Supportive care planning intervention}

A quality of life assessment completed by a nurse looking at physical, psychological, social and spiritual domains. This assessment leads to the development of a care plan

Case presentation at the multidisciplinary team meeting leading to recommendations tailored to patient need and referral to supportive care services.

Two nurse-administered education sessions on quality of life concerns

Content of physical and emotional well-being session

$$
\begin{aligned}
& \text { I) Pain/neuropathy } \\
& \text { I) Constipation/diarrhoea } \\
& \text { I) Fatigue } \\
& \text { 1) Appetite and weight loss } \\
& \text { I) Nausea/vomiting } \\
& \text { 》 Sleep disturbance } \\
& \text { ॥ Anxiety and depression }
\end{aligned}
$$

Content of social and spiritual well-being session

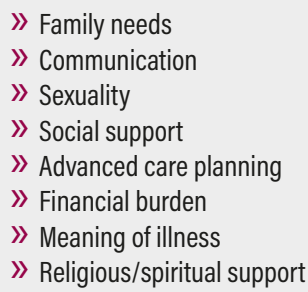

(Source: Sun et al 2016) 
Pain, constipation and lack of appetite/ weight loss were the most commonly selected topics in the physical and emotional wellbeing session, while social support, advanced care planning and family needs were the most commonly selected topics in the social and spiritual well-being session. The study reported that the intervention was feasible and acceptable (Sun et al 2016).

\section{Discussion}

There is a small, albeit developing, evidence base on the information, supportive and palliative care needs of people diagnosed with pancreatic cancer. They experience a range of physical and psychological needs that persist and change over time (Schildmann et al 2013, Beesley et al 2016a, Beesley et al 2016b, Hagensen et al 2016) which can make it difficult to maintain a good quality life (Gooden and White 2013, Beesley et al 2016a, Muircroft 2016). The literature suggests the main needs are pain control, fatigue, enzyme insufficiency and other dietary concerns, depression and anxiety. Interventions to support patients with these issues include integrated oncology and palliative care (Schenker et al 2018), holistic needs assessments (Macmillan Cancer Support 2013) and care plans (Polakowski et al 2015, Frick et al 2017). Interventions to support specific needs include early detection and referral to psychological support services and a multidisciplinary approach to the management of enzyme insufficiency and other dietary concerns (Gooden and White 2013, Muircroft 2016).

Beesley et al's (2016a) Australian study was the first quantitative assessment of the information needs of people diagnosed with pancreatic cancer. Research and literature reviews included in this review originate mainly in the US and Australia with no research from the UK. To address this Pancreatic Cancer UK is collaborating with Picker Institute Europe and Oxford Brookes University on a study of the care experiences and needs of people with pancreatic cancer in the UK. The study aims to explore the information and supportive care needs of this group, understand whether they feel they have all the information and support they need, and identify ways in which support services can be improved.

Further UK research exploring the effectiveness and suitability of supportive care interventions for people with pancreatic cancer is warranted, particularly as exploration of the effect of holistic needs assessments and survivorship care plans has focused on more common cancers, such as breast, colorectal, prostate and lung (Frick et al 2017). The move towards integrating supportive care in oncology is being explored in England through Enhanced Supportive Care (ESC), an NHS England initiative that aims to provide better and earlier access to symptom management during treatment and care. The programme is an all-cancer initiative exploring whether ESC can benefit people with cancer, including pancreatic cancer, to live better and longer (Berman et al 2017).

Recent NICE (2018) guidance recommends that a qualitative study should be undertaken to evaluate information and support interventions to address psychological needs of people with pancreatic cancer at different points in the care pathway. Research would help identify effective information and support interventions that would improve quality of life for people with pancreatic cancer and their family members/carers. Outcomes of interest would be quality of life, psychological wellbeing, ability to carry out normal activities and patient experience (NICE 2018).

\section{Implications for practice}

The role of the CNS is crucial to identifying patients' needs and coordinating their care (Macmillan Cancer Support 2013, Sun et al 2016). However, a range of healthcare professionals from across disciplines also need to be involved in the provision of supportive and palliative care to meet the complex needs of those with pancreatic cancer (Gooden and White 2013, Muircroft 2016). The importance of early referral to supportive and palliative care and the potential effectiveness and acceptability of integrated oncology, where oncology and palliative care teams work together, has been highlighted (Torgerson and Wiebe 2013, Beesley et al 2016b, Gooden et al 2016, Muircroft 2016, Schenker et al 2018). Beesley et al (2016a) suggest that the term 'palliative care' puts patients off accessing services, as it is associated with end of life and loss of hope, and propose the term 'supportive care' would be better.

Pain is a significant unmet need that must be managed more effectively (Grant and Wiegand 2011, Beesley et al 2016a) and pain specialists can support patients in this respect. Early detection of and support for anxiety and depression may positively affect quality of life, therefore access to counsellors and psychologists is vital (Muircroft 2016). Other allied healthcare professionals also have 
important roles to play in meeting patient needs: for example, dietitians can provide tailored support with managing dietary-related symptoms and dietary counselling on the best dosage and use of pancreatic enzymes to alleviate pancreatic exocrine insufficiency (Gooden and White 2013). Gooden et al (2016) refer to a 'whole team approach' where timely and appropriate discussions about supportive and palliative needs help patients and their families understand and manage their care.

\section{Conclusion}

People diagnosed with pancreatic cancer experience a high symptom burden that leads to extensive, ongoing and changing information, supportive and palliative care needs. The evidence suggests that needs are not adequately addressed, which affects current and future quality of life. For a disease with such a poor prognosis helping people maintain their quality of life is crucial. To date, identifying the needs of people with pancreatic cancer, and developing support services to meet those needs, is a neglected area of research and service provision and requires greater focus as has been the case for other cancer types.

It is essential that more is done to identify individual needs through regular assessment using tools such as holistic needs assessments. To meet people's needs it is important to explore and evaluate new models of care, such as early referral to supportive and palliative care and closer integration of oncology and palliative care. These practices may help people receive appropriate and timely supportive and palliative care to enable them to live as good a quality of life as possible for as long as possible.

\section{References}

Allemani C, Matsuda T, Di Carlo V et al (2018) Global surveillance of trends in cancer survival 2000-14 (CONCORD-3): analysis of individual records for 37 513025 patients diagnosed with one of 18 cancers from 322 population-based registries in 71 countries. Lancet. 391, 10125, 1023-1075

Beesley V, Janda M, Goldstein D et al (2016a) A tsunami of unmet needs: pancreatic and ampullary cancer patients' supportive care needs and use of community and allied health services. PsychoOncology. 25, 2, 150-157.

Beesley V, Wockner L, O'Rourke P et al (2016b) Risk factors for current and future unmet supportive care needs of people with pancreatic cancer. A longitudinal study. Supportive Care in Cancer. $24,8,3601-3599$.

Berman R, Elliott E, LaMola L et al (2017) 0-1 Enhanced supportive care in cancer. BMJ Supportive and Palliative Care. doi: org/10.1136/ bmispcare-2017-00133.1

Cancer Research UK (2018) Pancreatic Cancer Statistics. www.cancerresearchuk. org/health-professional/cancer-statistics/ statistics-by-cancer-type/pancreatic-cancer (Last accessed: 29 November 2018.)

Engebretson A, Matrisian L, Thompson C (2015) Pancreatic cancer: patient and caregiver perceptions on diagnosis, psychological impact, and importance of support. Pancreatology. $15,6,701-707$.
Freelove R, Walling A (2006) Pancreatic cancer: diagnosis and management. American Family Physician. 73, 3, 485-492.

Frick M, Vachani C, Hampshire M et al (2017) Survivorship after treatment of pancreatic cancer: insights via an internet-based survivorship care plan tool. International Journal of Radiation Oncology, Biology, Physics. 99, 2, E534-E535.

Gooden H, Kerry Tiller K, Mumford J et al (2016) Integrated psychosocial and supportive care needed for patients with pancreatic cancer. Cancer Forum. 40, 1,62-65.

Gooden H, White K (2013) Pancreatic cancer and supportive care - pancreatic exocrine insufficiency negatively impacts on quality of life. Supportive Care in Cancer. 21, 7, 1835-1841.

Grant M, Wiegand D (2011) Palliative care online: a pilot study on a pancreatic cancer website. Journal of Palliative Medicine. 14, 7, 846-851.

Grant M, Wiegand D (2013) Conversations with strangers. Journal of Hospice and Palliative Nursing $15,5,278-285$

Grant M, Wiegand D, Dy S (2015) Asking questions of a palliative care nurse practitioner on a pancreatic cancer website. Palliative and Supportive Care. 13,3,787-793.

Hagensen A, London A, Phillips J et al (2016) Using experience-based design to improve the care experience for patients with pancreatic cancer. Journal of Oncology Practice. 12, 12, 1035-1041.
Lee V, Cheng H, Li G et al (2012) Quality of life in patients with pancreatic cancer. Journal of the Pancreas. 13, 2, 182-184.

Lewis A, Pihlak R, McNamara M (2018) The importance of quality-of-life management in patients with advanced pancreatic ductal adenocarcinoma Current Problems in Cancer. 42, 1,26-39.

Macmillan Cancer Support (2013) The Recovery Package. www.macmillan.org.uk/ about-us/health-professionals/programmesand-services/recovery-package\#297633 (Last accessed: 29 November 2018.)

Melnyk B, Fineout-Overholt E (2011) EvidenceBased Practice in Nursing and Healthcare: A Guide to Best Practice. Lippincott, Williams and Wilkins, Philadelphia PA.

Muircroft W (2016) An Australasian perspective on the curative treatment of patients with pancreatic cancer, supportive care, and future directions for management. Ecancermedicalscience. 10, 700 .

National Institute for Health and Care Excellence (2018) Pancreatic Cancer in Adults: Diagnosis and Management. NICE Guideline NG85. Methods, Evidence and Recommendations. NICE, London.

Pancreatic Cancer UK (2016) Signs and Symptoms. www.pancreaticcancer.org.uk/informationand-support/facts-about-pancreatic-cancer/ signs-and-symptoms-of-pancreatic-cancer (Last accessed: 19 December 2018.
Polakowski T, Kerr M, Durand A (2015) Caring for the continuum of patients with pancreatic cance the importance of survivorship care planning. Clinical Journal of Oncology Nursing. 19, 1, E21-E24.

Quality Health (2016) National Cancer Patient Experience Survey National Reports 2016. www. ncpes.co.uk/reports/2016-reports/nationalreports-1 (Last accessed: 29 November 2018.)

Schenker Y, Bahary N, Claxton R et al (2018) A pilot trial of early specialty palliative care for patients with advanced pancreatic cancer: challenges encountered and lessons learned. Journal of Palliative Medicine. 21,1,28.

Schildmann J, Ritter P, Salloch S et al (2013) 'One also needs a bit of trust in the doctor ...' : a qualitative interview study with pancreatic cancer patients about their perceptions and views on information and treatment decision-making. Annals of Oncology. $24,9,2444-2449$.

Sun V, Ruel N, Chung V et al (2016) Pilot study of an interdisciplinary supportive care planning intervention in pancreatic cancer. Supportive Care in Cancer. 24, 8, 3417-3424.

Torgerson S, Wiebe L (2013) Supportive care of the patient with advanced pancreatic cancer. Oncology. 27, 3, 183-190. 